of globular structures, undergoes a transition to an unfolded form on heating, and in two of the states is protected against denaturation by specific inhibitors. (That is to say, the active centre remains intact.) There is a broad correspondence between these various states in the enzyme and zymogen, and in both cases phase diagrams have been constructed, which define the stability ranges of the various native and denatured forms with respect to $p \mathrm{H}$ and temperature.

The same authors (Delaage and Lazdunski, Biochem. Biophys. Res. Commun., 28, 390; 1967) have also examined the binding of calcium ions-which are known to have an influence on the activation of the zymogen by directing the hydrolysis of the correct peptide bond and suppressing adventitious cleavage. There is evidence that both possess a specific calciumbinding site, which is strong in one of the conformational states, weak in another and absent in the remaining two. The one state (that prevailing at neutral $p \mathbf{H}$ ) is therefore strongly stabilized by calcium, both with respect to transitions to other isomers, and denaturation by heat or urea. The observation that the site is still present after conversion of the zymogen to the enzyme suggests some rather extensive similarities of conformation. A second, and weaker, calcium binding site is present only in the zymogen, and is believed to lie in the peptide which is cleaved on activation.

A further study, of the interaction of trypsin with inhibitors and substrates, comes from D'Albis and Béchet (Biochim. Biophys. Acta, 140, 435; 1967), who find that interaction occurs even at relative extremes of $p \mathrm{H}$. This, using the approach of Delaage and Lazdunski, may presumably be seen in terms of the stabilization by the ligands of those isomeric states to which they bind. The curious observation is reported that one tyrosine residue becomes unavailable for titration when an inhibitor is present (in addition to the five already masked), until the protein is unfolded. This suggests a structural change on binding the inhibitor. There are also some changes in optical rotatory dispersion, which may be interpreted in terms of small conformation changes (in accordance with the "induced fit" notion). On the other hand, because the inhibitor contains a phenyl chromophore, which may well become optically active on binding, a new Cotton effect could also explain the result observed.

A further current article (Labouesse and Gervais, Europ. J. Biochem., 2, 215; 1967) clarifies a rather ambiguous situation, relating to the acetylation of trypsin and its effect on the enzymatic activity. Conditions have been established for the preparation of a derivative, in which all the $\varepsilon$-amino groups, but not the tyrosines or the $\alpha$-amino group, are acetylated. It is confirmed that both the lysines and tyrosines can be modified without destroying the activity, and indeed acetylation of tyrosines is found to enhance the esterolytic activity. The form in which only the $\varepsilon$-amino groups are acetylated has normal activity. The possible involvement of the terminal amino group in the active site remains unestablished.

\section{Beards and Bugs}

\section{from our Microbiology Correspondent}

A SOMEWHAT unusual piece of research has been reported recently by a team of mierobiologists working in the Industrial Health and Safety Office at Fort Detrick in Maryland. These workers (Barbeito, Mathews and Taylor, Appl. Microbiol., 15, 899; 1967) are concerned by the revival of beards among persons working with pathogenic micro-organisms and have investigated the hypothesis that a bearded man provides a risk of infection if his beard becomes contaminated in the laboratory. To test this hypothesis, a group of volunteers had their beards sprayed with cultures of non-infective Serratia marcescens and Bacillus subtilis var. niger, pigmented bacteria which are easily picked out in sampling programmes. With proper regard for their colleagues, Barbeito and coworkers used a bearded mannequin to study the transmission of Newcastle virus disease (Clostridium botulinum type A toxin) by beards, with chickens and guinea-pigs as test animals.

Sampling of contaminated beards was made by various means-swabs, membrane filters, agar impressions and saline rinses. In addition, beards were shorn and suspended in nutrient broth. Clean shaven subjects acted as controls and the effect of washing with a disinfectant soap was estimated for both groups. Comparison of the sampling procedures demonstrated that shorn beards blended in broth produced greater numbers of bacteria and that the other methods were likely to underestimate potential infection doses which may result from contact with unwashed beards. Here the investigators emphasize that several diseases are known for which the inhaled human infection dose is about ten microbes. Beards clearly held bacteria more effectively than skin even after soaping and rinsing. It is comforting to note, however, that in order to recover bacteria from unwashed beards it was necessary to apply populations of $10^{4}-10^{5}$, and laboratory contamination of this magnitude is unlikely to occur without the subject inhaling sufficient organisms to produce an illness. Thus beard-infection of close associates appears feasible only when the wearer has a recognizable accident or is subjected to regular doses of microbial aerosols, as might arise when carrying out a repetitive technique. On the other hand, results of experiments with NDV and the botulinum toxin indicate that contamination within the range of likely accidental beard contamination would create a serious human health hazard.

Interesting and salutary as the results of these trials are, one must keep in mind the fact that very many more people working with pathogens have hair on the top of their heads than on their faces and the issues raised in connexion with beards are surely more widely applicable. Clothing is another potential agency of dissemination and several reports have appeared on the persistence of micro-organisms, especially viruses, on clothing fabrics. To combat this, searches are being made for effective viricidal agents with which to impregnate fabrics. Sidwell, Dixon and McNeil (Appl. Microbiol., 15, 921; 1967) have illustrated the efficacy of treating fabrics with quaternary ammonium compounds or triazone resin in inactivating or greatly reducing the persistence of vaccinia and polio viruses on a variety of woollen and cotton materials. The treatment of fabrics to render them self-sanitizing is an attractive proposition, particularly when they are used in places liable to contamination by pathogenic micro-organisms. 